\title{
Results of Russian-Japanese Precise Timing of PSR $\mathrm{B} 1937+21$
}

\author{
Yu. P. Ilyasov ${ }^{1}$, M. Imae ${ }^{2}$, Y.Hanado ${ }^{2}$, V. V. Oreshko ${ }^{1}$, V. A. Potapov ${ }^{1}$, \\ A. E. Rodin ${ }^{1}$, M. Sekido ${ }^{2}$ \\ ${ }^{1}$ Pushchino Radio Astronomy Observatory, P. N. Lebedev Physical \\ Institute of the Russian Academy of Sciences, 142290 Pushchino, Russia \\ ${ }^{2}$ Communications Research Laboratory (CRL), 4-2-1 Nukui-kita, \\ Koganei, Tokio 184-8795, Japan
}

\begin{abstract}
Results of joint Russian-Japanese timing observations of the millisecond pulsar B1937+21 are presented. RMS of the post-fit residuals of the combined data is $1.8 \mu \mathrm{s}$. Secular change of dispersion measure with rate of $-0.0012 \pm 0.0003 \mathrm{pc} \mathrm{cm}^{-3}$ per year has been observed.
\end{abstract}

\section{Observations}

The millisecond pulsar B1937+21 has been observed at the Kalyazin Observatory since 1995 using the 64-m radio telescope and the multi-channel filterbank at $0.6 \mathrm{GHz}$ with $3.2 \mathrm{MHz}$ total bandwidth and two circular polarizations with $10 \mu \mathrm{s}$ time resolution in each channel (Ilyasov, Oreshko \& Poperechenko 1999; see also Ilyasov et al., these proceedins). The time standard of the observatory is based on a hydrogen maser and rubidium standards. Time comparison with UTC is made via GPS. Reduction of pulsar timing data is performed both with the TIMAPR software package developed at the PRAO (Doroshenko \& Kopeikin 1990), and with TEMPO (Taylor \& Weisberg 1989). Timing observations of the millisecond pulsar B1937+21 have been made at the Kashima Space Research Center (KSRC) at CRL since 1997 with the 34-m radio telescope at $2.15 \mathrm{GHz}$ and an acousto-optical device with total bandwidth $200 \mathrm{MHz}$ and time resolution of about $13 \mu \mathrm{s}$ (Hanado et al. 2002; see also Hanado et al., these proceedings).

\section{Data Reduction}

Fitting of joint data has been made with the TEMPO software. It has been observed that the dispersion measure (DM) varies in the direction of PSR B1937+21 (Kaspi, Taylor \& Ryba 1994). We have obtained two timing solutions: with constant DM, and with DM varying as a polynomial of up to 3rd order. All published results which contain some information about DM variations in the direction of $\mathrm{B} 1937+21$ were collected and together with our results are plotted in Figure 1. The residual RMS of the joint data is $1.8 \mu$ s after the DM corrections. 


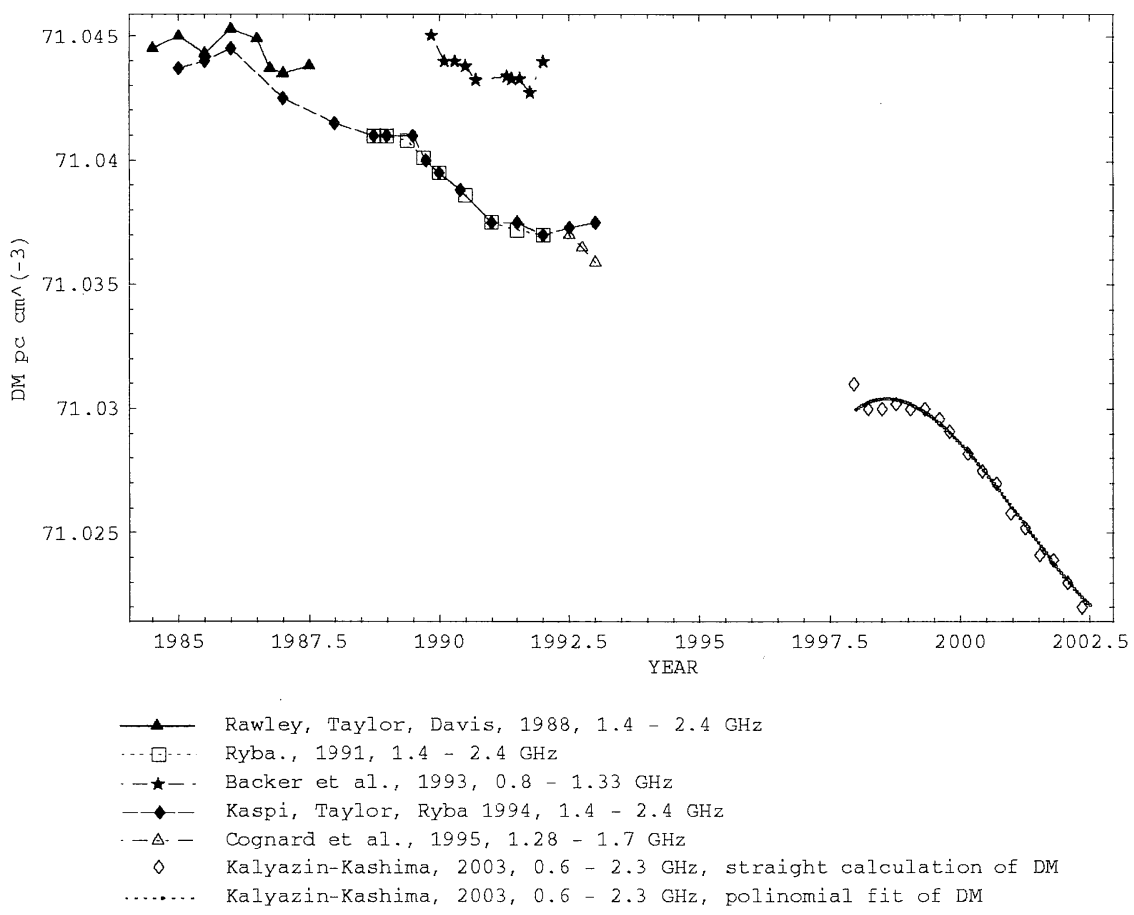

Figure 1. Slow changes in the DM of PSR B1937+21.

\section{Conclusion}

The pulsar B1937+21 has good long-term rotational stability. It can be considered as an independent precise reference clock in space for a Pulsar Timescale when two or more frequencies for pulsar timing are used (Draft new opinion ITU$\mathrm{R}$ [Doc.7/81], Timescale based on pulsar timing, 2002). We have shown that the DM of B1937+21 has continued to decrease, by $0.0012 \pm 0.0003 \mathrm{pc} \mathrm{cm}^{-3}$ per year during 1984-2002. Further long-term observations at widely separated frequencies are necessary to clarify the nature of the DM variations.

\section{References}

Doroshenko, O. V., \& Kopeikin, S. M. 1990, Sov. Astr., 34, 496

Hanado, Y., Shibuya, Y., Hosokawa, M., Sekido, M., Gotoh, T., \& Imae, M. 2002, PASJ, 54, 305

Ilyasov, Yu. P., Oreshko, V. V., Poperechenko, B. A. 1999, in Proc. of the Lebedev Physical Inst., Vol. 256

Kaspi, V. M., Taylor, J. H., \& Ryba, M. F. 1994, ApJ, 428, 713

Taylor, J. H., \& Weisberg, J. M. 1989, ApJ, 345, 434 